\title{
Stage IV Hodgkin's Disease Lymphocyte Predominance Type
}

National Cancer Institute

\section{Source}

National Cancer Institute. Stage IV Hodgkin's Disease Lymphocyte Predominance Type. NCl Thesaurus. Code C8831.

Stage IV adult Hodgkin disease means there is disseminated (multifocal) involvement of one or more extralymphatic organs, with or without associated lymph node involvement, or isolated extralymphatic organ involvement with distant (nonregional) nodal involvement. (from PDQ) 